\title{
Electronic multipoles in second harmonic generation and neutron scattering
}

\author{
G. van der Laan and S. W. Lovesey
}

\section{Published version information}

Citation: G van der Laan and SW Lovesey. "Electronic multipoles in second harmonic generation and neutron scattering." Phys Rev B 103, no. 12 (2021): 125124.

DOI: $\underline{10.1103 / P h y s R e v B .103 .125124}$

This version is made available in accordance with publisher policies. Please cite only the published version using the reference above. This is the citation assigned by the publisher at the time of issuing the APV. Please check the publisher's website for any updates.

This item was retrieved from ePubs, the Open Access archive of the Science and Technology Facilities Council, UK. Please contact epublications@stfc.ac.uk or go to http://epubs.stfc.ac.uk/ for further information and policies. 


\title{
Electronic multipoles in second harmonic generation and neutron scattering
}

\author{
G. van der Laan $\odot^{1}$ and S. W. Lovesey $\circledast^{1,2}$ \\ ${ }^{1}$ Diamond Light Source, Harwell Science and Innovation Campus, Didcot, Oxfordshire OX11 ODE, United Kingdom \\ ${ }^{2}$ ISIS Facility, STFC, Didcot, Oxfordshire OX11 0QX, United Kingdom
}

(Received 18 November 2020; revised 18 February 2021; accepted 18 February 2021; published 11 March 2021)

\begin{abstract}
Nonlinear optics, and particularly second harmonic generation (SHG), is increasingly used in many modern disciplines from material characterization in physical sciences to bioimaging in medicine and optical signal processing in information technology. We present a theoretical analysis yielding a strong estimate of the energyintegrated SHG response. Compact spherical multipoles are provided for the corresponding natural and magnetic circular dichroic signals. Like symmetry requirements in time and space are traced in the amplitude for magnetic neutron scattering, which includes all axial and polar (Dirac) contributions. Our method of working in terms of, now standard, electronic multipoles and Racah algebra, with full implementation of discrete symmetries, could be of use in a variety of other probes of matter.
\end{abstract}

DOI: 10.1103/PhysRevB.103.125124

\section{INTRODUCTION}

The study reported here contributes to the quest for a deeper understanding of intriguing, and potentially useful, electronic properties of substances. It focuses on what can be learned from the response of chiral and magnetic materials to their illumination by beams of photons or neutrons. To this end, we appeal to a formulation of response functions in terms of standard electronic multipoles. In the case of photon scattering, this innovation is made possible by integrating out intermediate degrees of freedom, which is accomplished by extensive use of Racah algebra. Following introductions to the two experimental techniques, suitable response functions are derived within an atomic framework. We demand compliance with fundamental physical properties of response functions that also must apply to results inferred from other experimental techniques, and not just photon and neutron scattering that are of immediate interest. Electronic degrees of freedom in the ground state are encapsulated in multipoles created from spherical tensor operators with discrete symmetries. Symmetries of space and time are embedded in a new identity for required matrix elements. Application of the identity to the second harmonic generation response has guided us to compact and precise results for natural circular and magnetic circular dichroism (NCD, MCD) that alluded us in a previous calculation. Specifically, we report closed expressions for NCD and MCD multipoles. Likewise, the identity underpins an amplitude for magnetic neutron scattering that includes conventional, axial magnetic dipoles and anapoles, and all allowed higher-order Dirac multipoles.

Nonlinear optical phenomena encompass physical processes originating from the interaction of light with matter, which modify the incoming electromagnetic field generating radiation of different frequency [1-3]. Such processes occur when a material interacts with intense light whereby its response yields fundamentally different properties than the one observed in the linear regime. Only after the introduction of the laser in 1960 [4], evidence of nonlinear phenomena was reported in 1961 by Franken et al. [5]. They detected the frequency doubling of radiation passing through a nonlinear crystal, the process of second harmonic generation in the visible light region, a phenomenon previously known for radio waves only. This observation is regarded as the beginning of the field of nonlinear optics, although two-photon absorption had already been predicted by Maria Göppert-Mayer in 1931 [6]. Armstrong et al. [7] and Loudon et al. [8] treated nonlinear optics within the general framework of response theory in 1962. More recent studies gathered from 142 publications, with an emphasis on molecular quantum electrodynamics, are reviewed by Andrews [9].

In this paper, we are primarily concerned with second harmonic generation (SHG), but results can be extended to other nonlinear effects. A simple picture to describe the SHG process is to consider a three-level system, depicted in Fig. 1. An incident photon excites an electron of the system, which is promoted to an empty state, and a second photon excites it to the next level. The state then de-excites to the equilibrium ground state under emission of a photon that, due to energy conservation, has twice the energy and frequency of the original photons. However, SHG is not just a three-step process, but instead a single three-body interaction. The intermediate states can be thought as virtual states described by many-body wave functions, subjected to microscopic interactions such as the Coulomb and exchange interactions and the spin-orbit coupling.

An important application of SHG is as a probe for spectroscopy or microscopy. As SHG excited by electric-dipole radiation is forbidden for a centrosymmetric medium, it is consequently highly sensitive to symmetry breaking. This makes it a selective probe for surfaces and interfaces of centrosymmetric media, where the bulk does not contribute and the frequency-doubled signal is therefore characteristic of the 


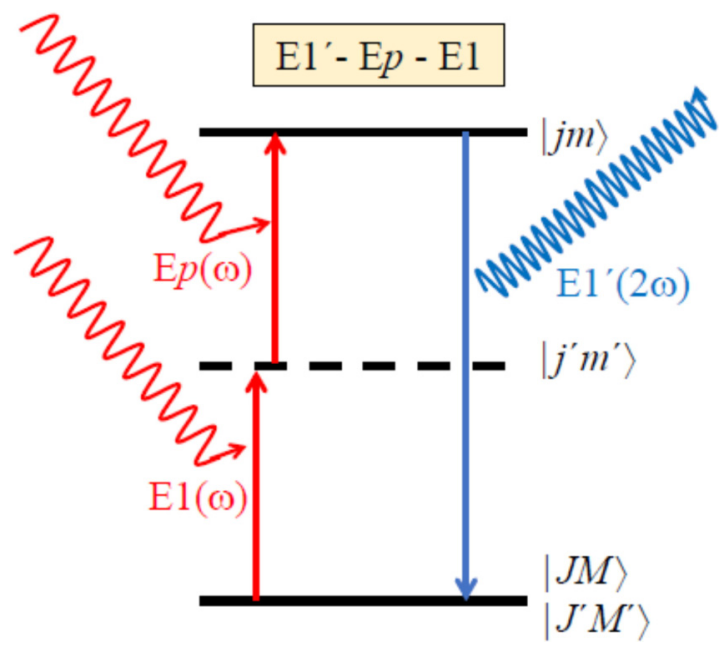

FIG. 1. Transitions that occur in second harmonic generation (frequency doubling) using three electronic operators $E 1^{\prime}-E p-E 1$, where $E p=E 1$ or $E 2$, and the primed operator $E 1^{\prime}$ relates to the secondary transition. Primary energy $E=\hbar \omega$ and integer $p$ labels modes available in the primary beam. Labeling of initial and final atomic states $\left|J^{\prime} M^{\prime}\right\rangle,|J M\rangle$ is used in Eqs. (1) and (3), together with intermediate states $|j m\rangle$ and $\left|j^{\prime} m^{\prime}\right\rangle$.

first few atomic layers close to the surface or interface. It also allows a time-resolved in situ monitoring of the surface reconstruction, of its chemistry, when molecules or other adsorbates are deposited [10]. This application ranges over a large variety of materials: metal surfaces, metal-electrolyte interfaces, semiconductors, oxides, insulator surfaces/interfaces, etc. [11,12]. SHG experiments have been frequently used to determine the average orientation of molecules adsorbed at surfaces, through measurements of the polarization dependence and phase [11]. In the last decade it has been affirmed as a selective nondestructive spectroscopy technique for the study of surfaces [13], superlattices [14-16], interfaces $[17,18]$ and two-dimensional materials [19]. Furthermore, second harmonic imaging microscopy has been employed to the study and imaging of cells and biological membranes, and especially collagen. It is a nondestructive probe that allows us to study in vivo biological systems in their environment [20-22].

At the x-ray region optical wave mixing was proposed nearly half a century ago as an atomic-scale probe of lightmatter interactions $[23,24]$. The recent advent of free-electron lasers (FELs) in the energy ranges from extreme ultraviolet to $\mathrm{x}$ rays allows us to explore these effects involving core-level resonances [25]. Yamamoto et al. [26] measured SHG at the $\mathrm{Fe} 3 p$ edge of gallium ferrate $\left(\mathrm{GaFeO}_{3}\right)$ using soft $\mathrm{x}$-ray FEL radiation. Other nonlinear optical techniques observed in the extreme-ultraviolet and $\mathrm{x}$-ray region include sum-frequency generation [27], four-wave mixing [28], and $\mathrm{x}$-ray two-photon absorption [29]. The ultrafast x-ray pulses from a high brightness $\mathrm{x}$-ray FEL provide the capability for time-resolved probing of atomic scale structure and electronic states in a material.

Similar to breaking of space-inversion symmetry, breaking of time-inversion symmetry by long-range magnetic ordering

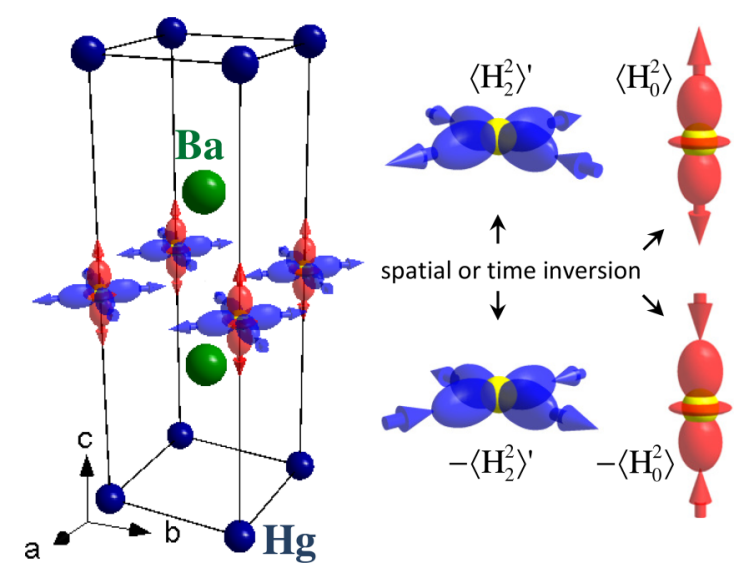

FIG. 2. Nonmagnetic $\mathrm{Hg} 1201$ is tetragonal and $\mathrm{Cu}$ ions occupy sites that are centers of spatial inversion symmetry. However, emergence of time-reversal violation in the pseudogap phase drives a reduction in $\mathrm{Cu}$ site symmetry that includes the loss of inversion symmetry. The magnetic state is epitomized by the condensation of Dirac quadrupoles $\left\langle H_{0}^{2}\right\rangle$ and $\left\langle H_{+2}^{2}\right\rangle^{\prime}$ defined in Eq. (14) [36].

or an applied magnetic field leads to additional contributions to SHG, which can be used to probe the magnetic structure [30,31]. Magnetic SHG is especially powerful in the case of antiferromagnets where, with a few specific exceptions, the usual magneto-optical methods fail because of the absence of bulk magnetization [32]. Rotation of the plane of polarization of reflected light (magneto-optic Kerr effect, often referred to by the acronym MOKE) is one direct manifestation of broken time-reversal symmetry. Despite the absence of bulk magnetization, magnetic crystal classes $m^{\prime} m^{\prime} m^{\prime}, 2 / m^{\prime}$ and $2 m^{\prime} m^{\prime}$ generate polarization rotation according Orenstein [33]. For these magnetic symmetries, the Kerr effect is mediated by magnetoelectric coupling, which can arise when antiferromagnetic order breaks inversion symmetry.

A direct observation of neutron diffraction by anapoles (Dirac dipoles) $[34,35]$ and compelling evidence from neutron Bragg diffraction patterns that ceramic superconductors support Dirac (magnetoelectric) quadrupoles [36] are reasons enough to revisit theories of neutron scattering. The onset of magnetic Bragg diffraction in ceramic superconductors occurs at temperatures associated with the appearance of the pseudogap state as determined by probes such as angle-resolved photoemission spectroscopy, NMR, and optical conductivity [37]. By and large, theories of neutron scattering in widespread use simply rely on conventional, axial dipoles and, even then, ignore orbital magnetism.

Bragg diffraction experiments have demonstrated that underdoped $\mathrm{HgBa}_{2} \mathrm{CuO}_{4+\delta}(\mathrm{Hg} 1201)$ samples possess magnetic order indexed on the chemical structure, and the same is found in identical experiments on a structurally more complicated cuprate $\mathrm{YBa}_{2} \mathrm{Cu}_{3} \mathrm{O}_{6+x}(\mathrm{YBCO})$, with two $\mathrm{CuO}_{2}$ plaquettes in a unit cell $[38,39]$. A ferro-type motif of Dirac quadrupoles, depicted in Fig. 2, is consistent with all available neutron diffraction data [36]. The story for $\mathrm{Hg} 1201$ is similar to YBCO, where neutron magnetic Bragg diffraction and the Kerr effect have been observed and successfully related to Dirac quadrupoles [33]. While $\mathrm{Hg} 1201$ and YBCO 
possess the same magnetic space group $\left(C m^{\prime} m^{\prime} m^{\prime}\right.$, magnetic crystal-class $m^{\prime} m^{\prime} m^{\prime}$ ), not surprisingly, there are significant differences in the symmetries at sites used by $\mathrm{Cu}$ ions. Notably, $\mathrm{Cu}$ ions in nonmagnetic $\mathrm{Hg} 1201$ occupy sites that are centers of spatial inversion symmetry. However, condensation of Dirac quadrupoles in the pseudogap phase breaks the inversion symmetry.

Use of electronic multipoles to discuss Bragg diffraction of $\mathrm{x}$ rays and neutrons is absolutely standard. Our use of them here to formulate response functions for SHG and neutron scattering has previously proved useful in several other photon scattering processes. Examples include x-ray magneto-optical sum rules [40-42], a unified formulation of dichroic signals using the Borrmann effect and twisted photon beams [43], resonant $\mathrm{x}$-ray diffraction from chiral electric-polarization structures [44], and a demonstration that superchiral photons reveal magnetic circular dichroism [45]. But, our innovation for SHG starts by finding a representation for the response in terms of a spherical tensor operator. We have already accomplished this task with extensive use of Racah algebra, and we refer the reader to a previous publication for additional details [46]. Beyond are superior results for NCD and MCD, reported in Sec. II, in terms of simple operator equivalents for multipoles. These reveal the nature of the electronic entities that are probed, and form a basis for future specific calculations. As with all atomic calculations, it is necessary to specify adopted conventions, and this we fulfill with two appendixes. Material therein is utilized in a formulation of the magnetic neutron scattering amplitude based on our new identity for required matrix elements. Our findings are reported in Sec. III and Appendix C.

\section{SECOND HARMONIC GENERATION RESPONSE}

By way of an introduction to our formulation of the SHG response, we turn to the amplitude of x-ray scattering enhanced by an electric dipole-electric dipole (E1-E1) event derived from the Kramers-Heisenberg dispersion formula. In standard form it is not a response function represented by a spherical tensor to which Racah algebra can be applied. A spherical tensor operator, by which we mean one that obeys the Wigner-Eckart Theorem, emerges after intermediate degrees of freedom are integrated out. Similar reasoning is used in our treatment of the SHG response, and it follows pioneer work by Judd and Ofelt $[47,48]$. The quantity of interest in the dispersion formula is $\left\{\left\langle l J M\left|\boldsymbol{\varepsilon}^{\prime} \cdot \mathbf{R}\right| \lambda j m\right\rangle\left\langle\lambda j m|\boldsymbol{\varepsilon} \cdot \mathbf{R}| l^{\prime} J^{\prime} M^{\prime}\right\rangle\right\}$, where $\boldsymbol{\varepsilon}^{\prime}(\boldsymbol{\varepsilon})$ is the purely real photon polarization vector for the secondary (primary) absorption process, and $\mathbf{R}$ is the electronic dipole operator. Virtual intermediate states have atomic quantum numbers $\lambda \mathrm{jm}$. If these are treated as spherically symmetric the dyadic can be replaced by its value integrated over projections $m$ in an application of the formula. Concomitant with neglect of angular anisotropy, energies of the intermediate states also are taken to be weak functions of the projections, and they will not contribute to our energy- integrated signal. One finds [49]

$$
\begin{gathered}
\sum_{m}\left\{\left\langle l J M\left|\boldsymbol{\varepsilon}^{\prime} \cdot \mathbf{R}\right| \lambda j m\right\rangle\left\langle\lambda j m|\boldsymbol{\varepsilon} \cdot \mathbf{R}| l^{\prime} J^{\prime} M^{\prime}\right\rangle\right\} \\
\propto \sum_{K, Q}(-1)^{Q} X_{-Q}^{K}\left\langle J M\left|T_{Q}^{K}\right| J^{\prime} M^{\prime}\right\rangle
\end{gathered}
$$

where the spherical tensor $\mathbf{X}^{K}$ is a sole function of polarization vectors, and $\left\langle J M\left|T_{Q}^{K}\right| J^{\prime} M^{\prime}\right\rangle$ derived from dipole operators satisfies Eq. (A5), whereupon $\mathbf{T}^{K}$ can be used as a true spherical tensor operator. Intermediate orbital angular momentum $\lambda$ is a parameter in the corresponding reduced matrix element (RME), cf. Appendixes A and B.

Components of $\mathbf{X}^{K}$ are evaluated from Eq. (A4) after setting $j=j^{\prime}=1$ to represent simple vectors, while the Clebsch-Gordan coefficient [50,51] embodies the triangle rule for addition of vectors to form spherical tensors $K=0$, 1 and $2 ; X_{0}^{0}=-(1 / \sqrt{3})\left(\boldsymbol{\varepsilon}^{\prime} \cdot \boldsymbol{\varepsilon}\right), \mathbf{X}^{1}=\left\{(i / \sqrt{2})\left(\boldsymbol{\varepsilon}^{\prime} \times \boldsymbol{\varepsilon}\right)\right\}$, and the traceless quadrupole $\mathbf{X}^{2}$ has a diagonal component $X_{0}^{2}=$ $\left\{(1 / \sqrt{6})\left(3 \varepsilon_{z}^{\prime} \varepsilon_{z}-\boldsymbol{\varepsilon}^{\prime} \cdot \boldsymbol{\varepsilon}\right)\right\}$. The RME for $\mathbf{T}^{K}$ is purely real and thus identified with $Z\left(l J, l J^{\prime}\right)$ in Eq. (B5). One finds that $\left(l J\left\|T^{K}\right\| l J^{\prime}\right)$ is a sum on $a$ (spin) and $b$ (orbital) of a standard unit tensor $W^{(a, b) K}\left(l J, l J^{\prime}\right)$ restricted by the condition $(a+b+K)$ even. The unit tensor for one electron is defined in Eq. (C8). The simple result $W^{(a, b) K}\left(l J, l J^{\prime}\right)=$ $(-1)^{J^{\prime}-J} W^{(a, b) K}\left(l J^{\prime}, l J\right)$ is a direct consequence of said condition. Therefore, the RME in Eq. (B5) is proportional to $\chi[1+$ $\left.\chi^{2}\right]$, and $\chi^{2}=\left\{(-1)^{K} \sigma_{\theta}\right\}$ for a parity-even event. In conclusion, the electronic multipole for an E1-E1 absorption event is different from zero when $(-1)^{K}=\sigma_{\theta}$, meaning the dipole is time-odd (magnetic) and the monopole and quadrupole are time-even (chargelike). Identical results can be derived from crossing-symmetry [49]. The results might be inferred from the observation that $\mathbf{X}^{0}$ and $\mathbf{X}^{2}$ differ in phase from $\mathbf{X}^{1}$ by $90^{\circ}$.

Electric quadrupole-electric quadrupole (E2-E2) enhancement is similarly described by Eq. (1) on replacing the dipole operator therein by $[(\boldsymbol{\varepsilon} \cdot \mathbf{R})(\mathbf{q} \cdot \mathbf{R})]$ where $\mathbf{q}$ is the primary photon wave vector, and likewise its secondary equivalent $\left(\boldsymbol{\varepsilon} \cdot \mathbf{q}=\boldsymbol{\varepsilon}^{\prime} \cdot \mathbf{q}^{\prime}=0\right)$. According to the triangle rule for addition of two tensors of rank 2, E2-E2 scattering is described by five electronic multipoles $K=0, \ldots, 4$. Not surprisingly, their time signature $\sigma_{\theta}=(-1)^{K}$. Beyond electric parity-even absorption considered thus far is parity-odd $E 1-E 2$ and, also, electric dipole-magnetic dipole $(E 1-M 1)$ events [49]. Scattering amplitudes for these events are sums of multipoles that are time even $\left(\sigma_{\pi} \sigma_{\theta}=-1\right)$ and time odd $\left(\sigma_{\pi} \sigma_{\theta}=+1\right)$. Using dipoles by way of illustrations, these are expectation values of a displacement $\left\langle R_{Q}\right\rangle$ and an anapole (toroidal moment), respectively.

$S H G$ response. A Cartesian form of natural circular dichroism (NCD) from the SHG response uses an $E 1^{\prime}-E 1-E 1$ event. Our coordinates are defined by the primary beam parallel to the $z$ axis and $\sigma$ polarization parallel to the $x$ axis. The secondary beam, engaged in an $E 1$ event, is inclined to the $z$ axis and its polarization vector has a component parallel to it. The generic form of the energy-integrated NCD signal is (see Ref. [52] and Sec. A 6 of Ref. [46]),

$$
F(\mathrm{NCD}) \propto P_{2} \sum_{f, f^{\prime}}\left\{\langle g|x| f\rangle\left\langle f|y| f^{\prime}\right\rangle\left\langle f^{\prime}|z| g\right\rangle-\langle g|y| f\rangle\left\langle f|x| f^{\prime}\right\rangle\left\langle f^{\prime}|z| g\right\rangle\right\} .
$$


Here, $g$ denotes the electronic ground state and $f$ and $f^{\prime}$ label intermediate states, while $P_{2}$ is a pseudoscalar for helicity in the primary radiation. We proceed as in Eq. (1) and integrate out intermediate projections of the orbital angular momentum, with the same caveat about our energy-integrated signal not being a function of intermediate energies, i.e., they are independent of labels $f$ and $f^{\prime}$, to a good approximation. Again, products of matrix elements factor, as depicted in Fig. 3, although the analog of the photon tensor $\mathbf{X}^{K}$ is more complicated [46]. Our goal is to produce operator equivalents for multipoles in NCD and magnetic circular dichroism (MCD), i.e., $F(\mathrm{NCD})$ and $F(\mathrm{MCD})$, defined by corresponding response RMEs that we derive in accord with Eq. (B5).

Specifically, a result for $Z^{K}\left(l J, l^{\prime} J^{\prime}\right)$ for use in Eq. (B5) suitable for NCD and MCD in the SHG response is calculated from $\left\{\langle l J M|E 1| \lambda j m\rangle\left\langle\lambda j m|E p| \lambda^{\prime} j^{\prime} m^{\prime}\right\rangle\left\langle\lambda^{\prime} j^{\prime} m^{\prime}|E 1| l^{\prime} J^{\prime} M^{\prime}\right\rangle\right\}$, where index $p=1,2$, and 3 labels modes available in the primary beam. Previously, we established that $p=1$ (2) for NCD (MCD). Following nontrivial sums on projections $m$ and $m^{\prime}$ [Eq. (11) in Ref. [46]],

$$
\begin{aligned}
& Z^{K}\left(l J, l^{\prime} J^{\prime}\right) \\
& =(-1)^{J-j}(l J\|\mathbf{R}\| \lambda j)\left(\lambda j\left\|\mathbf{C}^{p}(\mathbf{R})\right\| \lambda^{\prime} j^{\prime}\right)\left(\lambda^{\prime} j^{\prime}\|\mathbf{R}\| l^{\prime} J^{\prime}\right) \\
& \quad \times\left\{\begin{array}{lll}
1 & p & K \\
J^{\prime} & J & j
\end{array}\right\}\left\{\begin{array}{lll}
p & 1 & p \\
J^{\prime} & j & j^{\prime}
\end{array}\right\},
\end{aligned}
$$

with remaining sums on $j, j^{\prime}, \lambda, \lambda^{\prime}$ understood. The normalized spherical harmonic $\mathbf{C}^{1}(\mathbf{R})=\mathbf{R}$. (Strictly speaking, the

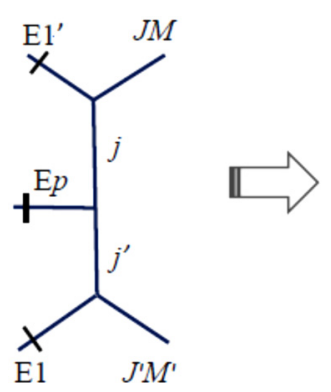

SHG

Matrix element

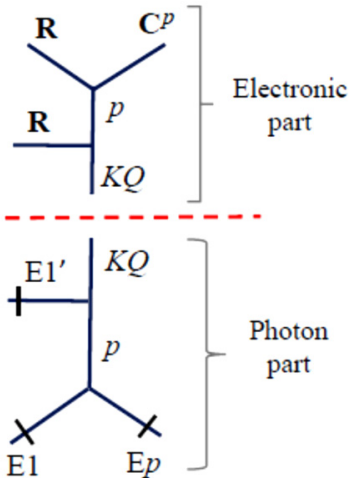

Recoupled

Matrix element
FIG. 3. Integrating out projections of intermediate states in the matrix element of the $E 1^{\prime}-E p-E 1$ event, depicted in Fig. 1, creates an electronic spherical tensor operator with rank $K$, projection $Q$ and an RME given by Eq. (3). Photon and electronic tensors are linked by a mode label $p$, which has no analogy in two-photon events described by the Kramers-Heisenberg dispersion formula.

spatial argument is the unit vector $\mathbf{n}=\mathbf{R} / R$, whereas our use of $\mathbf{R}$ for the dipole operator might help visual tracking of the development.) The corresponding RME is purely real with $\left(l j \| \mathbf{C}^{k}|| l^{\prime} j^{\prime}\right)=(-1)^{j-j^{\prime}}\left(l^{\prime} j^{\prime} \| \mathbf{C}^{k}|| l j\right)$ in the general case. Derivation of $Z^{K}\left(l^{\prime} J^{\prime}, l J\right)$ from Eq. (3) is thereafter straightforward. Another recoupling of variables in Eq. (3) leads to an entirely new RME for the SHG response that is consistent with Eq. (B5). It has an appealing form:

$$
\begin{aligned}
\left(l J\left\|\mathcal{O}^{K}\right\| l^{\prime} J^{\prime}\right)= & \chi(-1)^{J-j} \sum_{x}(2 x+1)\left\{\begin{array}{lll}
1 & 1 & x \\
p & K & p
\end{array}\right\}\left\{\begin{array}{lll}
1 & 1 & x \\
j & j^{\prime} & p \\
J & J^{\prime} & K
\end{array}\right\}\left[1+\sigma_{\pi} \sigma_{\theta}(-1)^{x+p}\right] \\
& \times(l J\|\mathbf{R}\| \lambda j)\left(\lambda j\left\|\mathbf{C}^{p}(\mathbf{R})\right\| \lambda^{\prime} j^{\prime}\right)\left(\lambda^{\prime} j^{\prime}\|\mathbf{R}\| l^{\prime} J^{\prime}\right) .
\end{aligned}
$$

The Racah $6 j$ and $9 j$ symbols in Eqs. (3) and (4) are defined in accord with standard references [50,51], which are sources of their many symmetry properties. Both dichroic signals of interest have $\sigma_{\pi} \sigma_{\theta}=-1$, and the index $x=0,1,2$ in Eq. (4) is uniquely defined, namely, $x=2$ for $\operatorname{NCD}(p=1, K=2)$ and $x=1$ for $\operatorname{MCD}(p=2, K$ odd).

In order to perform sums on $j$ and $j^{\prime}$ in Eq. (4) we need an explicit result for the RME of a spherical harmonic $\left(l j\left\|\mathbf{C}^{k}\right\| l^{\prime} j^{\prime}\right)$. In our chosen $s-l$ coupling scheme [51],

$$
\left(\sigma l j\left\|\mathbf{C}^{k}\right\| \sigma l^{\prime} j^{\prime}\right)=(-1)^{\sigma+l^{\prime}+j+k}\left[(2 j+1)\left(2 j^{\prime}+1\right)\right]^{1 / 2}\left\{\begin{array}{lll}
l & j & \sigma \\
j^{\prime} & l^{\prime} & k
\end{array}\right\}\left(l\left\|\mathbf{C}^{k}\right\| l^{\prime}\right)
$$

with

$$
\left(l\left\|\mathbf{C}^{k}\right\| l^{\prime}\right)=(-1)^{l}\left[(2 l+1)\left(2 l^{\prime}+1\right)\right]^{1 / 2}\left(\begin{array}{ccc}
l & k & l^{\prime} \\
0 & 0 & 0
\end{array}\right)
$$

and the $3 j$ symbol can be different from zero for $\left(l+k+l^{\prime}\right)$ even.

We define the response RME $\left(l \| \mathcal{O}^{K}|| l^{\prime}\right)$ to conform with an identical $s$ - $l$ coupling scheme, i.e., $\left(l J \| \mathcal{O}^{K}|| l^{\prime} J^{\prime}\right)$ and $\left(l \| \mathcal{O}^{K}|| l^{\prime}\right)$ are related as in Eq. (5), with

$$
\left(l\left\|\mathcal{O}^{K}\right\| l^{\prime}\right)=-\chi \sum_{x}(2 x+1)\left\{\begin{array}{lll}
1 & 1 & x \\
p & K & p
\end{array}\right\}\left\{\begin{array}{lll}
l & l^{\prime} & K \\
1 & 1 & x \\
\lambda & \lambda^{\prime} & p
\end{array}\right\}\left[1+\sigma_{\pi} \sigma_{\theta}(-1)^{x+p}\right](l\|\mathbf{R}\| \lambda)\left(\lambda\left\|\mathbf{C}^{P}(\mathbf{R})\right\| \lambda^{\prime}\right)\left(\lambda^{\prime}\|\mathbf{R}\| l^{\prime}\right) .
$$

Equation (6), with sums on intermediate angular momenta $\lambda, \lambda^{\prime}$, is our core result for the SHG response, from which we proceed to derive NCD and MCD signals. 
The NCD signal in the SHG response is created by

$$
\left(l\left\|\mathcal{O}^{2}(\mathrm{NCD})\right\| l^{\prime}\right)=-\chi \sqrt{(2 / 15)}\left(l\left\|\left\{\mathbf{R} \otimes \mathbf{C}^{2}\right\}^{2}\right\| l^{\prime}\right),
$$

and $\chi^{2}=-1$. Equation (7) follows from summation over angular momenta $\lambda, \lambda^{\prime}$ with $p=1$, which is appropriate for NCD. A standard definition of a tensor product in Eq. (7) is modeled on Eq. (A4),

$$
\left\{\mathbf{A}^{a} \otimes \mathbf{B}^{b}\right\}_{Q}^{K}=\sum_{\alpha, \beta} \mathbf{A}_{\alpha}^{a} \mathbf{B}_{\beta}^{b}(a \alpha b \beta \mid K Q) .
$$

Equation (7) says that the NCD signal in the SHG response can be calculated using matrix elements of the operator $\left\{\mathbf{R} \otimes \mathbf{C}^{2}\right\}^{2}$, which is evidently parity odd and time even. Simply put, the multipole $\left\langle\left\{\mathbf{R} \otimes \mathbf{C}^{2}\right\}^{2}\right\rangle$ for the material under illumination is allowed to be different from zero when NCD is detected. As in our previous calculation, the operator equivalent is a dyadic [46]. The index $p=2$ for the MCD signal and another lengthy mathematical exercise yields

$$
\begin{aligned}
\left(l\left\|\mathcal{O}^{K}(\mathrm{MCD})\right\| l^{\prime}\right)= & -\chi[6 /(2 K+1)]^{1 / 2} \\
& \times\left\{\begin{array}{lll}
1 & 1 & 1 \\
2 & K & 2
\end{array}\right\}\left(l\left\|\left\{\mathbf{C}^{2} \otimes \mathbf{L}\right\}^{K}\right\| l^{\prime}\right),
\end{aligned}
$$

with $K=1$ and 3, and $\chi^{2}=+1$. The operator equivalent $\left\{\mathbf{C}^{2} \otimes \mathbf{L}\right\}^{K}$ is manifestly both parity even and time odd. Multipoles $\left\langle\left\{\mathbf{C}^{2} \otimes \mathbf{L}\right\}^{K}\right\rangle$ calculated with Eq. (9) are used in the MCD signal $F(\mathrm{MCD})=\left\{q_{0} P_{2}\left[\sqrt{2}\left\langle\mathcal{O}_{0}^{1}\right\rangle+\sqrt{3}\left\langle\mathcal{O}_{0}^{3}\right\rangle\right]\right\}$, where $q_{0} \equiv q_{z}$ represents the primary wave vector [46]. For a crystalline material, permitted motifs of $\left\langle\left\{\mathbf{R} \otimes \mathbf{C}^{2}\right\}^{2}\right\rangle$ and $\left\langle\left\{\mathbf{C}^{2} \otimes \mathbf{L}\right\}^{K}\right\rangle$ are delineated by time and space in the unit cell, and prescribed by the relevant space group, likewise for symmetry in reduced dimensions and molecular systems.

\section{NEUTRON SCATTERING}

The amplitude for magnetic scattering of neutrons is written $\mathbf{Q}_{\perp}=[\boldsymbol{\kappa} \times(\mathbf{Q} \times \boldsymbol{\kappa})]$ using a unit wave vector $\boldsymbol{\kappa}=\mathbf{k} / k$ where $\mathbf{k}$ is the scattering wave vector. An intermediate operator can be written

$$
\mathbf{Q}=\exp \left(i \mathbf{R}_{j} \cdot \mathbf{k}\right)\left[\mathbf{s}_{j}-(i / \hbar k)\left(\boldsymbol{\kappa} \times \mathbf{p}_{j}\right)\right],
$$

in which $\mathbf{R}_{j}, \mathbf{s}_{j}$, and $\mathbf{p}_{j}$ are operators for position, spin, and linear momentum of unpaired electrons, respectively. Note that $\mathbf{Q}$ is arbitrary to within any function proportional to $\boldsymbol{\kappa}$.

Orbital-spin contribution. If $\mathbf{k}$ is treated as a small parameter,

$$
\{\exp (i \mathbf{R} \cdot \mathbf{k}) \mathbf{s}\}=\mathbf{s}+i(\mathbf{R} \cdot \mathbf{k}) \mathbf{s}-\cdots .
$$

The leading term contributes to an overused approximation for $\mathbf{Q}$, namely, $\mathbf{Q} \approx(\mathbf{L}+2 \mathbf{S}) / 2$ for an isolated ion with orbital angular momentum. As for the second, manifestly parity-odd term in Eq. (11) there is a standard decomposition,

$$
\begin{aligned}
i(\mathbf{R} \cdot \mathbf{k}) \mathbf{s}_{\alpha}= & (k R) i \kappa_{\beta}\left\{(1 / 3) \delta_{\alpha \beta} \mathbf{s} \cdot \mathbf{n}+(1 / 2) \varepsilon_{\alpha \beta \gamma}(\mathbf{s} \times \mathbf{n})_{\gamma}\right. \\
& \left.+(1 / 2)\left[\mathbf{s}_{\alpha} \mathbf{n}_{\beta}+\mathbf{s}_{\beta} \mathbf{n}_{\alpha}-(2 / 3) \delta_{\alpha \beta} \mathbf{s} \cdot \mathbf{n}\right]\right\}, \quad(12)
\end{aligned}
$$

where $\mathbf{n}=\mathbf{R} / R$ (Einstein summation convention). The scalar operator does not contribute to $\mathbf{Q}_{\perp}$, i.e., the electronic Dirac

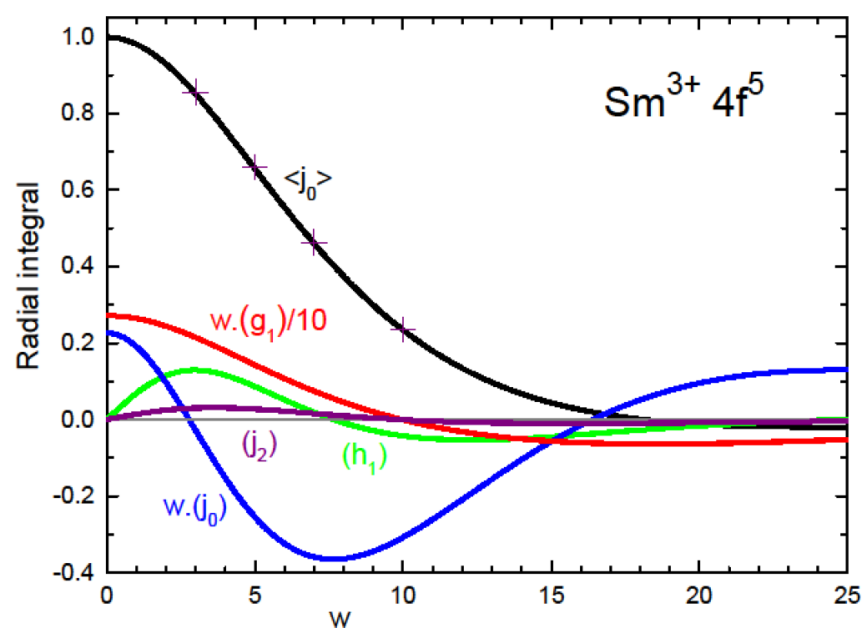

FIG. 4. Radial integrals for Dirac multipoles. Dimensionless variable $w=12 \pi a_{\mathrm{o}} s$, where $a_{\mathrm{o}}$ is the Bohr radius, while the standard variable for radial integrals $s$ is derived from the Bragg angle and neutron wavelength $s=\sin (\theta) / \lambda$. Legend: red: $\left[w \times\left(g_{1}\right)\right] / 10$, green: $\left(h_{1}\right)$, blue: $\left[w \times\left(j_{0}\right)\right]$ and purple: $\left(j_{2}\right)$. Note that $\left(g_{1}\right)$ and $\left(j_{0}\right)$ from Eq. (C3) arise from the component of $\mathbf{Q}$ in Eq. (10) that contains the linear momentum operator and they are proportional to $1 / w$ as the wave vector approaches zero. Atomic wave functions are $4 f^{5}-5 d^{1}$. Also included in the figure is the standard radial integral $\left\langle j_{0}(k)\right\rangle$. Results obtained with our $\mathrm{Sm}^{3+}\left(4 f^{5}\right)$ wave function are denoted by the continuous black curve, to which we added for comparison four values $(+)$ derived from the standard interpolation formula [53].

monopole is not visible in neutron scattering, although it is visible in resonance enhance x-ray scattering [49]. Next in line, the spin anapole contribution $\left\{\kappa_{\beta} \varepsilon_{\alpha \beta \gamma}(\mathbf{s} \times \mathbf{n})_{\gamma}\right\}=$ $[\boldsymbol{\kappa} \times(\mathbf{s} \times \mathbf{n})]_{\alpha}$ has been unambiguously detected in diffraction by two compounds with the $\mathrm{C} 15$ cubic Laves structure [34,35]. Last, the traceless Dirac quadrupole accounts for Bragg diffraction patterns collected on pseudogap phases of Hg1201 and YBCO [36].

For arbitrary magnitudes of $\mathbf{k}$ we make use of a purely real function $\left(h_{K}\right)$ that is the mean value of the spherical Bessel function $j_{K}(k R)$ with respect to radial parts of atomic orbitals. It reduces to a standard radial integral $\left\langle j_{K}(k)\right\rangle$ for equivalent electrons [53]; Fig. 4 contains $\left(h_{1}\right)$ and $\left\langle j_{0}(k)\right\rangle$ for samarium, and definitions are derived from Eq. (C4). The integer $K$ is even for equivalent electrons, and odd for orbitals with opposing parities. We go on to find

$$
\{\exp (i \mathbf{R} \cdot \mathbf{k}) \mathbf{s}\}=\sum_{K, K^{\prime}} i^{K^{\prime}+1}(2 K+1)^{1 / 2}\left\{\mathbf{C}^{K}(\boldsymbol{\kappa}) \otimes \mathbf{H}^{K^{\prime}}\right\}^{1} .
$$

The RME of the tensor operator,

$$
\begin{aligned}
\mathbf{H}^{K^{\prime}}= & (-i)^{K^{\prime}+K+1}\left[(2 K+1)\left(2 K^{\prime}+1\right) / 3\right]^{1 / 2} \\
& \times\left(h_{K}\right)\left\{\mathbf{s} \otimes \mathbf{C}^{K}(n)\right\}^{K^{\prime}},
\end{aligned}
$$

satisfies the fundamental requirement Eq. (B5). To this end, $\quad\left(l J\left\|\left\{\mathbf{s} \otimes \mathbf{C}^{K}(\mathbf{n})\right\}^{K^{\prime}}\right\| l^{\prime} J^{\prime}\right)=\{\sqrt{(3 / 2)}$ $\left.\left(l\left\|\mathbf{C}^{K}\right\| l^{\prime}\right) W^{(1, K) K^{\prime}}\left(l J, l^{\prime} J^{\prime}\right)\right\} \quad$ with $\quad\left(K+l+l^{\prime}\right)$ even, $K^{\prime}=K, K \pm 1$ and a maximum rank $K^{\prime}=\left(1+l+l^{\prime}\right)$. Equation (12) is recovered from (13) and (14) using 
$j_{1}(k R) \approx(k R) / 3$ for a small argument, and $K^{\prime}=1$ and 2 . Values of the unit tensor for equivalent electrons are tabulated [54]. The rank $K^{\prime}$ is odd if electrons belong to a manifold $J=J^{\prime}$, which can be verified from (C8) for a single electron and states $l=l^{\prime}$.

Orbital contribution. An analog of the spin dipole in Eq. (11),

$$
\begin{aligned}
& -(i / \hbar k)\{\exp (i \mathbf{R} \cdot \mathbf{k})(\boldsymbol{\kappa} \times \mathbf{p})\} \\
& \quad=\left\{(1 / 2)\left[\left\langle j_{0}(k)\right\rangle+\left\langle j_{2}(k)\right\rangle\right] \mathbf{L}+i(\boldsymbol{\kappa} \times \mathbf{D})\right\}+\cdots,
\end{aligned}
$$

uses a Dirac dipole $\mathbf{D}=(1 / 2)\left[i\left(g_{1}\right) \mathbf{n}-\left(j_{0}\right) \boldsymbol{\Omega}\right]$, where $\boldsymbol{\Omega}$ is an orbital anapole defined in Eq. (C5), and integrals $\left(j_{0}\right)$ and $\left(g_{1}\right)$ are made with radial functions belonging to states with opposing parities, e.g., atomic $4 f$ and $5 d$ states. Unlike
Eq. (11), Eq. (15) is valid for arbitrary values of the wave vector. While $\left\langle j_{0}(0)\right\rangle=1$ for equivalent electrons, $\left(j_{0}\right)$ and $\left(g_{1}\right)$, depicted in Fig. 4, both diverge as $k$ approaches zero.

An RME for the orbital operator Eq. (15) that includes all tensor operators is complicated, as illustrated by the comparatively simple result for equivalent electrons,

$$
-\{\exp (i \mathbf{R} \cdot \mathbf{k})(\boldsymbol{\kappa} \times \nabla)\}=\sum_{K^{\prime}}\left\{\mathbf{C}^{K^{\prime}-1}(\boldsymbol{\kappa}) \otimes \mathrm{O}^{K^{\prime}}\right\}^{1},
$$

with odd $K^{\prime}=1,3, \ldots(2 l-1)$, and a tensor operator defined by the RME,

$$
\left(l\left\|O^{K^{\prime}}\right\| l\right)=k A\left(K^{\prime}, l\right)\left[\left\langle j_{K^{\prime}-1}(k)\right\rangle+\left\langle j_{K^{\prime}+1}(k)\right\rangle\right],
$$

where the purely real function,

$$
\begin{aligned}
A\left(K^{\prime}, l\right)= & i^{K^{\prime}-1}\left[K^{\prime}\left(K^{\prime}+1\right)-2(l+1)\right]^{-1}\left(l\left\|\left\{\mathbf{L} \otimes \mathbf{C}^{K^{\prime}}(\mathbf{n})\right\}^{K^{\prime}}\right\| l+1\right) \\
& \times\left\{\left[K^{\prime}\left(2 K^{\prime}-1\right)\left(2 K^{\prime}+1\right)(2 l+1)\left(2 l+2+K^{\prime}\right)\left(2 l+1-K^{\prime}\right)\right] /[3(2 l+3)]\right\}^{1 / 2},
\end{aligned}
$$

uses definition (C3). The RME $\left(l J \| O^{K^{\prime}}|| l J^{\prime}\right)=$ $\left\{\sqrt{2}\left(l\left\|O^{K^{\prime}}\right\| l\right) W^{\left(0, K^{\prime}\right) K^{\prime}}\left(l J, l J^{\prime}\right)\right\}$ complies with Eqs. (B1) and (B3), given $\sigma_{\pi} \sigma_{\theta}=-1\left(\sigma_{\theta}=-1, \sigma_{\pi}=+1\right.$, ) and $K^{\prime}$ odd. One finds $A(1, l)=(l|| L|| l) / 2$, consistent with Eq. (15). Results Eqs. (16)-(18) are precisely Eq. (11.48) in Ref. [55].

Contributions to the Dirac dipole $\mathbf{D}$ and all higher-order operators are discovered in an expression suitable for atomic states with opposing parities $l \neq l^{\prime}$. It is delegated to Appendix $\mathrm{C}$ on account of its complexity. One thing to note here is that the result Eq. (C2) is an explicit example of the response RME in Eq. (B5).

\section{DISCUSSION AND CONCLUSIONS}

Optical radiation can be expanded in multipole terms, such as electric dipole $(E 1)$, electric quadrupole (E2), and magnetic dipole $(M 1)$, together with higher-order terms that can normally be neglected. In a two-photon process, described by the Kramer-Heisenberg formulism, the optical activity induced by a pure transition must have even parity, so that only interference terms can give odd parity. The pure $E 1-E 1$ process produces magnetic circular dichroism (MCD), which is a time-odd, parity-even event. The E1-M1 interference in the visible region allows natural circular dichroism (NCD) or optical rotation in powdered samples or in solution and single crystals. In the case of $\mathrm{x}$-ray absorption, which involves core to valence shell excitations, $M 1$ transitions are forbidden due to the restriction imposed by the monopole selection rule for the radial part. Although in the visible region the E2 transitions are negligibly small, their magnitude increases with photon energy, so that for harder $\mathrm{x}$ rays the $E 1-E 2$ interference becomes significant, giving rise to NCD.

In this paper, we extended our inquiry to the second harmonic generation (SHG) response, with two primary photons of frequency $\omega$ and one secondary photon of frequency $2 \omega$, as depicted in Fig. 1. Instead of the Kramer-Heisenberg formulism, SHG is adequately described by third-order perturbation theory, cf. Eq. (2). Using theoretical techniques from atomic physics we derived explicit expressions for electronic multipoles of the SHG response. Polarization-dependent photon spectroscopy (dichroism) of the SHG response is shown to reveal chiral and magnetic properties of a sample. Two dichroic signals are allowed with electric-dipole $(E 1)$ and electric-quadrupole (E2) scattering events, and both require circular polarization in the primary beam. NCD is derived from an $\left(E 1^{\prime}-E 1-E 1\right)$ event, and a parity-even event $\left(E 1^{\prime}-E 2\right.$ $E 1)$ yields MCD [46].

Results Eqs. (7) and (9) for the SHG response are energyintegrated signals. That is to say, from Eq. (7), the multipole $\left\langle\left\{\mathbf{R} \otimes \mathbf{C}^{2}(\mathbf{R})\right\}^{2}\right\rangle$ is the total NCD signal available from a substance in a suitably designed measurement, with a like statement derived from Eq. (9) for the MCD signal. Here, $\{. \otimes .\}^{K}$ denotes a tensor product of rank $K, \mathbf{C}^{2}(\mathbf{R})$ is a spatial spherical harmonic of rank 2 normalized such that $\mathbf{C}^{1}(\mathbf{R})=\mathbf{R}$, and the MCD signal is a sum of multipoles $\left\langle\left\{\mathbf{C}^{2}(\mathbf{R}) \otimes \mathbf{L}\right\}^{K}\right\rangle$ with $K=1$ and 3. A meaningful context for our results is the analogy between Eqs. (7) and (9) with celebrated sum rules for conventional, parity-even dichroic signals [40-42] and their extensions to parity-odd signals [56], derived from the Kramers-Heisenberg dispersion formula. In the present setting, Eqs. (7) and (9) are products of our new statement in Eq. (B5) for the reduced matrix element (RME) of a response function. While compatible with earlier results for the same quantities, we submit that they are stronger statements. This claim is grounded on the recoupling Eq. (4) of the exact result Eq. (3) for matrix elements in an $E 1^{\prime}-E p-E 1$ event, depicted in Fig. 1, that was not previously accomplished [46]. Notably, we now complete sums over intermediate states and arrive at closed expressions for NCD and MCD multipoles cited above. A straightforward passage to results in Eqs. (7) and (9) is testament to insight from the recoupling.

A proof that $\left(E 1^{\prime}-M 1-M 1\right)$ with $\mathbf{M}=(\mathbf{L}+2 \mathbf{S})$ does not produce a NCD signal in the SHG response was given in a previous paper (Appendix B in Ref. [46]). In the present setting, the null result for NCD follows most easily from Eq. (3), because RMEs for spin and orbital angular momentum are diagonal with respect to angular momentum, e.g., 
$\left(\sigma \lambda^{\prime}\|\mathbf{S}\| \sigma l^{\prime}\right)$ and $\left(\sigma \lambda^{\prime}\|\mathbf{L}\| \sigma l^{\prime}\right)$ are zero for $\lambda^{\prime} \neq l^{\prime}$. One finds mixed contributions from $\mathbf{S}$ and $\mathbf{L}$ to $Z^{K}\left(l J, l^{\prime} J^{\prime}\right)$ sum to zero. On the other hand, pure $\mathbf{S}$ and pure $\mathbf{L}$ contributions to $Z^{K}\left(l J, l^{\prime} J^{\prime}\right)$ are nonzero but each one cancels out in the response RME $\left(l J \| \mathcal{O}^{K}|| l^{\prime} J^{\prime}\right)$ found in Eq. (B5).

Turning to the second experimental technique in the present study, the orbital contribution to the magnetic neutron scattering amplitude can be calculated with Eq. (17). The equation, combined with (18), finds immediate application for a single electron in an atomic shell, e.g., $\mathrm{Ce}^{3+}$ [57]. An extension of Eq. (17) to equivalent electrons is well established and, therefore, not pursued here [54,55]. The generalization to electrons in different atomic orbitals given in Appendix $\mathrm{C}$ is a specific example of our new statement in Eq. (B5) for the RME of a response function. The orbital-spin contribution to the magnetic scattering amplitude Eq. (14), by comparison, is a much simpler response function that obeys Eq. (B5), as expected-perhaps deceptively simple, since Eq. (14) is the sole source of even rank multipoles in the scattering amplitude that are permitted by a correlation of the spin anapole and spatial degrees of freedom $[54,58]$.

\section{ACKNOWLEDGMENT}

Figure 2 was prepared by Dr. D. D. Khalyavin.

\section{APPENDIX A: DEFINITIONS}

Cartesian $(x, y, z)$ and spherical components $R_{Q}$ of a vector $\mathbf{R}$ are related by $x=\left(R_{-1}-R_{+1}\right) / \sqrt{2}, y=i\left(R_{-1}+\right.$ $\left.R_{+1}\right) / \sqrt{2}, z=R_{0}$. Evidently, spherical components of a dipole are complex for $Q$ different from zero, and $\left\{R_{Q}\right\}^{\times}=$ $(-1)^{Q} R_{-Q}$, where ${ }^{\times}$is complex conjugation. Operators we use possess discrete symmetries with respect to the reversal of the coordinates of space and time, with corresponding signatures $\sigma_{\pi}$ and $\sigma_{\theta}$. An operator is parity even (odd) for $\sigma_{\pi}=+1$ $(-1)$, while time even (odd) for $\sigma_{\theta}=+1(-1)$. Parity and time inversions are conjugate operations that reverse the sign of a four-vector. The vector (dipole) $\mathbf{R}$ is polar with $\sigma_{\pi}=-1$, whereas spin $\mathbf{S}$ (and orbital angular momentum $\mathbf{L}$ and $\mathbf{J}=$ $\mathbf{S}+\mathbf{L}$ ) is axial with $\sigma_{\pi}=+1$. We adopt the convention that $\sigma_{\theta}$ is the sign difference between time-reversal and complex conjugation. Since the two operations applied separately to $R_{Q}$ yield identical results, the time signature $\sigma_{\theta}=+1$, while $S_{Q}$ possesses a time signature $\sigma_{\theta}=-1$. More generally, the rank of a spherical tensor operator is labeled by a positive integer $K$, and it possesses $(2 K+1)$ projections $-K \leqslant Q \leqslant K$. Our operators obey $\left[\mathcal{O}_{Q}^{K}\right]^{+}=(-1)^{Q} \mathcal{O}_{-Q}^{K}$, where ${ }^{+}$denotes Hermitian conjugation, and the diagonal component $\mathcal{O}_{0}^{K}$ is Hermitian.

The action of our time-reversal operator $\theta$ on a timedependent wave function of the system $\psi(\mathbf{R}, t)$ is $\theta \psi(\mathbf{R}, t)=$ $\psi^{\times}(\mathbf{R},-t)$, while $\theta \psi(\mathbf{H})=\psi^{\times}(\mathbf{H})=\psi(-\mathbf{H})$ for a stationary state subject to a magnetic field $\mathbf{H}$. A matrix element of an arbitrary operator $B$ satisfies [59]

$$
(\psi, B \varphi)=\left(\theta \varphi, \overline{\mathrm{B}}^{+} \theta \psi\right)
$$

with $\bar{B}=\theta B \theta^{-1}$, which follows from standard properties $\theta(c \psi)=c^{\times}(\theta \psi)$ and $(\theta \psi, \theta B \varphi)=(\psi, B \varphi)^{\times}(c$ is a classical number), whereupon

$$
(\psi, B \varphi)_{\mathbf{H}}=\left(\varphi, \bar{B}^{+} \psi\right)_{-\mathbf{H}}=\sigma_{\theta}(\varphi, B \psi)_{-\mathbf{H}},
$$

and our definition $\bar{B}=\sigma_{\theta} B^{+}$equates change in the polarity of an applied magnetic field with change in the mean value of $B$ with respect to the reversal of time, which is a sensible outcome.

The Wigner $3 j$ symbol and Clebsch-Gordan coefficient $(a \alpha b \beta \mid K Q)$ are related by

$$
(a \alpha b \beta \mid K Q)=(-1)^{-a+b-Q} \sqrt{(2 K+1)}\left(\begin{array}{lll}
a & b & K \\
\alpha & \beta & -Q
\end{array}\right) .
$$

The $3 j$ symbol is invariant with respect to inversion of spatial coordinates $[49,50]$. The parity operator commutes with the rotation operator, because rotations are generated by parity-even operators $J_{\alpha}$. Thus, states $|J M\rangle$ that differ only in projections $M$ have the same parity. The time-reversal operator $\theta$ changes the sign of a projection. If we equate $\theta(c|J M\rangle)$ with $c^{\times}(-1)^{J-M}|J,-M\rangle$, a composite state,

$$
|J M\rangle=\sum_{m m^{\prime}}|j m\rangle\left|j^{\prime} m^{\prime}\right\rangle\left(j m j^{\prime} m^{\prime} \mid J M\right),
$$

is fully compatible with it. Notably, $\theta^{2}|J M\rangle=(-1)^{2 J}|J M\rangle=$ $\pm|J M\rangle$, where the upper sign is for integer $J$ and the lower sign is for half-integer $J$.

The Wigner-Eckart theorem says a matrix element $\left(J M, \mathcal{O}_{Q}^{K} J^{\prime} M^{\prime}\right) \equiv\left\langle J M\left|\mathcal{O}_{Q}^{K}\right| J^{\prime} M^{\prime}\right\rangle$ is related to a reduced matrix element (RME) that does not depend on projections, $M$ and $M^{\prime}$. We use a standard definition of the theorem whereby

$$
\left\langle J M\left|\mathcal{O}_{Q}^{K}\right| J^{\prime} M^{\prime}\right\rangle=(-1)^{J-M}\left(\begin{array}{lll}
J & K & J^{\prime} \\
-M & Q & M^{\prime}
\end{array}\right)\left(J\left\|\mathcal{O}^{K}\right\| J^{\prime}\right) .
$$

The RME $\left(J \| \mathcal{O}^{K}|| J^{\prime}\right)$ is also called a double-barred matrix element. Total angular momentum $J=(l \pm \sigma)$, with $\sigma=$ $1 / 2$, where $l$ labels the orbital angular momentum. Electronic matrix elements and RMEs are diagonal with respect to the magnitude of the spin, of course, and $\sigma$ is not made explicit henceforth.

\section{APPENDIX B: RESPONSE FUNCTIONS}

Two key identities must be satisfied in the RME of a response function. The first is a straightforward consequence of the definition $\left[\mathcal{O}_{Q}^{K}\right]^{+}=(-1)^{Q} \mathcal{O}_{-Q}^{K}$ and the Wigner-Eckart theorem, namely (Secs. A 3 and A 5 in Ref. [51]),

$$
\left(l J\left\|\mathcal{O}^{K}\right\| l^{\prime} J^{\prime}\right)=(-1)^{J-J^{\prime}}\left(l^{\prime} J^{\prime}\left\|\mathcal{O}^{K}\right\| l J\right)^{\times},
$$

and it is worth noting that $(-1)^{J-J^{\prime}}=(-1)^{J^{\prime}-J}$. The second identity is a direct outcome of (A1) for which we now use an operator of rank $K$ with parity signature $\sigma_{\pi}$, i.e., application of the parity operator results in $\left(P_{\pi} B^{K} P_{\pi}^{-1}\right)=\sigma_{\pi} B^{K}$. Simultaneous inversion of space and time coordinates on an elemental state $|J M\rangle$ is represented by

$$
P_{\pi} \theta(c|J M\rangle)=c^{\times}(-1)^{J-M}|J,-M\rangle,
$$

whereupon [51]

$$
\left(l^{\prime} J^{\prime}\left\|B^{K}\right\| l J\right)=(-1)^{J^{\prime}-J}(-1)^{K} \sigma_{\pi} \sigma_{\theta}\left(l J\left\|B^{K}\right\| l^{\prime} J^{\prime}\right)
$$


follows from the Wigner-Eckart theorem. Together, Eqs. (B1) and (B3) yield

$$
\left(l J\left\|\mathcal{O}^{K}\right\| l^{\prime} J^{\prime}\right)=(-1)^{K} \sigma_{\pi} \sigma_{\theta}\left(l J\left\|\mathcal{O}^{K}\right\| l^{\prime} J^{\prime}\right)^{\times} .
$$

A fundamental result for the RME of a response function that sits at the heart of all we report combines Eqs. (B1) and (B3). It is simple to verify that

$$
\left(l J\left\|\mathcal{O}^{K}\right\| l^{\prime} J^{\prime}\right)=\chi\left[Z^{K}\left(l J, l^{\prime} J^{\prime}\right)+\rho Z^{K}\left(l^{\prime} J^{\prime}, l J\right)\right]
$$

obeys requirements Eqs. (B1) and (B3) for purely real $Z^{K}\left(l J, l^{\prime} J^{\prime}\right)$. The two phase factors are found to be $\chi^{2}=$ $\left\{(-1)^{K} \sigma_{\pi} \sigma_{\theta}\right\}$ and $\rho=\chi^{2}(-1)^{J^{\prime}-J}$, meaning $\rho= \pm 1$ and $\chi$ purely real or imaginary.

\section{APPENDIX C: NEUTRON SCATTERING AMPLITUDE}

Referring to Eq. (15), it is natural to separate the electronic linear momentum operator $\mathbf{p}=-i \hbar \nabla$ into its angular and radial components that we label by the letters $a$ and $r$. It can be shown that [60]

$$
\begin{gathered}
-(1 / \hbar k) \exp (\mathrm{i} \mathbf{R} \cdot \mathbf{k})(\boldsymbol{\kappa} \times \nabla)_{q} \\
=\sum_{K, K^{\prime}}\left\{\mathbf{C}^{K}(\boldsymbol{\kappa}) \otimes \mathbf{O}^{K^{\prime}}(K ; a)\right\}_{q}^{1} \\
+\sum_{K^{\prime}}\left\{\mathbf{C}^{K^{\prime}}(\boldsymbol{\kappa}) \otimes \mathbf{O}^{K^{\prime}}(r)\right\}_{q}^{1}
\end{gathered}
$$

The radial tensor operator vanishes for equivalent electrons, as we will see, unlike the angular tensor (17) for $l=l^{\prime}$. The angular tensor in (C1) necessarily depends on two indices, $K$ and $K^{\prime}$, while a single index (rank) suffices for the radial tensor

Angular tensor

$$
\begin{aligned}
\mathbf{O}^{K^{\prime}}(K ; a)= & i^{K+K^{\prime}-1}(2 K+1) \sqrt{\left[3\left(2 K^{\prime}+1\right)\right]} \sum_{x, y}(-1)^{(1+K+x) / 2}\left(j_{x}\right)(2 x+1)(2 y+1) \\
& \times\left(\begin{array}{ccc}
1 & K & x \\
0 & 0 & 0
\end{array}\right)\left(\begin{array}{ccc}
1 & x & y \\
0 & 0 & 0
\end{array}\right)\left\{\begin{array}{lll}
1 & x & y \\
K^{\prime} & 1 & 1
\end{array}\right\}\left\{\begin{array}{lll}
K & K^{\prime} & 1 \\
1 & 1 & x
\end{array}\right\}\left\{\mathbf{L} \otimes \mathbf{C}^{y}(\mathbf{n})-(-1)^{K^{\prime}+y} \mathbf{C}^{y}(\mathbf{n}) \otimes \mathbf{L}\right\}^{K^{\prime}},
\end{aligned}
$$

with $\mathbf{n}=\mathbf{R} / R$. In Eq. (C2),

$$
\left(l^{\prime}\left\|\left\{\mathbf{L} \otimes \mathbf{C}^{y}(\mathbf{n})\right\}^{K^{\prime}}\right\| l\right)=(-1)^{l+l^{\prime}}\left(l\left\|\left\{\mathbf{C}^{y}(\mathbf{n}) \otimes \mathbf{L}\right\}^{K^{\prime}}\right\| l^{\prime}\right)=(-1)^{K^{\prime}+l+l^{\prime}} \sqrt{2 K^{\prime}+1}\left(l^{\prime}\|L\| l^{\prime}\right)\left(l^{\prime}\left\|\mathbf{C}^{y}\right\| l\right)\left\{\begin{array}{lll}
l^{\prime} & l & K^{\prime} \\
y & 1 & l^{\prime}
\end{array}\right\} .
$$

The first thing to note is that $\left(l\left\|\mathbf{O}^{K^{\prime}}(K ; a)\right\| l^{\prime}\right)=$ $(-1)^{K^{\prime}+1}\left(l^{\prime}\left\|\mathbf{O}^{K^{\prime}}(K ; a)\right\| l\right)$ follows from $(\mathrm{C} 2)$ and $\left(y+l+l^{\prime}\right)$ even. In consequence, the RME satisfies Eq. (B5). The radial integral is

$$
\left(j_{x}\right)=\int_{0}^{\infty} d R R^{2} f_{l}(R) f_{l^{\prime}}(R)\left\{j_{x}(k R) / k R\right\},
$$

with $j_{x}(k R)$ a spherical Bessel function, and $f_{l}(R)$ and $f_{l^{\prime}}(R)$ radial parts of electron orbitals labeled by their angular momenta $l$ and $l^{\prime}$.

The rank $y$ defines the parity of the tensor operator, i.e., $\sigma_{\pi}=(-1)^{y}$. It is even for equivalent electrons in an atomic shell, $l=l^{\prime}$, and $\mathbf{O}^{K^{\prime}}(K ; a)$ together with $(\mathrm{C} 1)$ are identical to Eqs. (16)-(18). However, it is worthwhile to add a few words about properties of Eq. (C2) for equivalent electrons and recovery of the dipole contribution displayed in Eq. (15). Use of Eq. (C3) in Eq. (C2) shows that the tensor operator is different from zero for $\left(y+K^{\prime}\right)$ odd, meaning $K^{\prime}$ odd. Moreover, $x=K^{\prime}$ on inspection of Eq. (C2). The sum on $K$ in Eq. (C1) is removed by addition of a function proportional to $\kappa_{q}$ that leaves the amplitude for magnetic scattering of neutrons $\mathbf{Q}_{\perp}$ unchanged. The outcome is to set $K=K^{\prime}-1$ in Eq. (C1), and simultaneously replace all dependence on $K$ by $\sqrt{\left\{\left(2 K^{\prime}-1\right) /\left[3\left(K^{\prime}+1\right)\right]\right\}}$ in Eq. (C2). It remains to perform the sum on $y=K^{\prime} \pm 1$, and it is proportional to $(l|| L|| l)$ for $K^{\prime}=x=1$ with a constant of proportionality that reproduces Eq. (15). The combination of radial integrals seen in Eq. (17) follows from the identity $j_{n}(z)=\left\{z\left[j_{n-1}(z)+j_{n+1}(z)\right] /(2 n+\right.$ $1)\}$.
Consideration of orbitals with angular momenta that differ by an odd integer equates to parity $y$ odd. It follows that $x$ is even and $K$ is odd in Eq. (C2). One finds $y=K=K^{\prime}$ for $K^{\prime}$ odd, while $x=K^{\prime}$ for $K^{\prime}$ even. To recover the Dirac dipole $\mathbf{D}=-\left\{(1 / 2)\left(j_{0}\right) \boldsymbol{\Omega}\right\}$ contribution to Eq. (15), set $K^{\prime}=1$ and $y=K=1$. Thereafter, two uses of an identity for the tensor product of two dipoles. First $\left\{\mathbf{C}^{1}(\boldsymbol{\kappa}) \otimes \mathbf{O}^{1}\right\}^{1}=(i / \sqrt{2})(\boldsymbol{\kappa} \times$ $\left.\mathbf{O}^{1}\right)$ in Eq. (C1), and the same identity yields

$$
\begin{aligned}
& \left\{\mathbf{L} \otimes \mathbf{C}^{1}(\mathbf{n})-\mathbf{C}^{1}(\mathbf{n}) \otimes \mathbf{L}\right\}^{1} \\
& \quad=(i / \sqrt{2})[\mathbf{L} \times \mathbf{n}-\mathbf{n} \times \mathbf{L}]=(i / \sqrt{2}) \boldsymbol{\Omega} .
\end{aligned}
$$

Properties of the orbital anapole $\boldsymbol{\Omega}$ are reviewed in Ref. [49].

It remains to define the radial tensor operator in Eq. (C1) and survey its properties. The radial integral in the result,

$$
\begin{aligned}
\left(l\left\|\mathbf{O}^{K^{\prime}}(r)\right\| l^{\prime}\right)= & i^{K^{\prime}}(1 / 2)\left(g_{K^{\prime}}\right)^{l, l^{\prime}}\left[K^{\prime}\left(K^{\prime}+1\right)\left(2 K^{\prime}+1\right) / 3\right]^{1 / 2} \\
& \times\left(l\left\|\mathbf{C}^{K^{\prime}}(\mathbf{n})\right\| l^{\prime}\right), \quad(\text { C6) }
\end{aligned}
$$

is odd with respect to the exchange of $l$ and $l^{\prime}$. Specifically,

$$
\begin{aligned}
\left(g_{K^{\prime}}\right)^{l, l^{\prime}}= & \left(2 K^{\prime}+1\right) \int_{0}^{\infty} d R R^{2}\left[f_{l}(R)(d / d R) f_{l^{\prime}}(R)\right. \\
& \left.-f_{l^{\prime}}(R)(d / d R) f_{l}(R)\right]\left\{j_{K^{\prime}}(k R) /\left(R k^{2}\right)\right\}
\end{aligned}
$$

and $\left(g_{1}\right)^{l, l^{\prime}}$ is included in Fig. 4. The RME in Eq. (C6) satisfies Eqs. (B1) and (B3). Eq. (C6) evaluated for $K^{\prime}=1$, using 
$\mathbf{C}^{1}(\mathbf{n})=\mathbf{n}$, yields the contribution made to the Dirac dipole. Note that the contribution to $\mathbf{D}$ uses the anti-Hermitian, timeodd and parity-odd operator $\{i \mathbf{n}\}$. The RME of the momentum operator is

$$
\left(l\|\mathbf{p}\| l^{\prime}\right)=(\hbar / 2)\left[\left(l\|\boldsymbol{\Omega}\| l^{\prime}\right)-2 i\left(l\|\mathbf{n}\| l^{\prime}\right)\right] .
$$

We can infer from this result that the anapole and $\{i \mathbf{n}\}$ possess identical discrete symmetries.
The unit tensor for one electron [51],

$$
\begin{aligned}
W^{(a, b) K^{\prime}}\left(\sigma l j, \sigma l^{\prime} j^{\prime}\right)= & {\left[(2 j+1)\left(2 K^{\prime}+1\right)\left(2 j^{\prime}+1\right)\right]^{1 / 2} } \\
& \times\left\{\begin{array}{ccc}
\sigma & \sigma & a \\
l & l^{\prime} & b \\
j & j^{\prime} & K^{\prime}
\end{array}\right\},
\end{aligned}
$$

with $\sigma=1 / 2$, and $j=(l \pm \sigma)$. The magnitude of the $9 j$ symbol is unchanged by an even or odd exchange of columns or rows, but an odd exchange changes its sign by a factor $(-1)^{\Re}$ with $\Re=\left(1+a+l+l^{\prime}+b+j+j^{\prime}+K^{\prime}\right)[50,51]$.
[1] N. Bloembergen, Nonlinear Optics (Benjamin, New York, 1965).

[2] Y. R. Shen, The Principles of Nonlinear Optics (WileyInterscience, New York, 1984).

[3] R. D. Boyd, Nonlinear Optics, 3rd ed. (Academic, London, 2008).

[4] T. Maiman, Nature (London) 187, 493 (1960).

[5] P. A. Franken, A. E. Hill, C. W. Peters, and G. Weinreich, Phys. Rev. Lett. 7, 118 (1961).

[6] M. Göppert-Mayer, Ann. Phys. (Leipzig) 401, 273 (1931).

[7] J. A. Armstrong, N. Bloembergen, J. Ducuing, and P. S. Pershan, Phys. Rev. 127, 1918 (1962).

[8] R. Loudon, Proc. Phys. Soc. 80, 952 (1962).

[9] D. L. Andrews, Symmetry 10, 298 (2018).

[10] U. Höfer, Appl. Phys. A 63, 533 (1996).

[11] R. M. Corn and D. A. Higgins, Chem. Rev. 94, 107 (1994).

[12] G. Lüpke, Surf. Sci. Rep. 35, 75 (1999).

[13] M. Kauranen, T. Verbiest, J. J. Maki, and A. Persoons, J. Chem. Phys. 101, 8193 (1994).

[14] B. Fluegel, A. Mascarenhas, J. F. Geisz, and J. M. Olson, Phys. Rev. B 57, R6787 (1998).

[15] C. Zhang, X. Xiao, N. Wang, K. K. Fung, and M. M. T. Loy, Appl. Phys. Lett. 72, 2072 (1998).

[16] E. Ghahramani, D. J. Moss, and J. E. Sipe, Phys. Rev. B 43, 8990 (1991).

[17] V. I. Gavrilenko, Phys. Rev. B 77, 155311 (2008).

[18] A. Savoia, D. Paparo, P. Perna, Z. Ristic, M. Salluzzo, F. M. Granozio, U. S. di Uccio, C. Richter, S. Thiel, J. Mannhart, and L. Marrucci, Phys. Rev. B 80, 075110 (2009).

[19] Y. Wang, J. Xiao, S. Yang, Y. Wang, and X. Zhang, Opt. Mater. Express 9, 1136 (2019).

[20] P. J. Campagnola, H. A. Clark, W. A. Mohler, A. Lewis, and L. M. Loew, Nat. Biotech. 21, 1356 (2003).

[21] B. E. Cohen, Nature (London) 467, 407 (2010).

[22] H. M. Su, J. T. Ye, Z. K. Tang, and K. S. Wong, Phys. Rev. B 77, 125428 (2008).

[23] I. Freund and B. F. Levine, Phys. Rev. Lett. 25, 1241 (1970).

[24] P. M. Eisenberger and S. L. McCall, Phys. Rev. A 3, 1145 (1971).

[25] M. Beye, R. Y. Engel, J. O. Schunck, S. Dziarzhytski, G. Brenner, and P. S. Miedema, J. Phys.: Condens. Matter 31, 014003 (2019).

[26] S. Yamamoto, T. Omi, H. Akai, Y. Kubota, Y. Takahashi, Y. Suzuki, Y. Hirata, K. Yamamoto, R. Yukawa, K. Horiba,
H. Yumoto, T. Koyama, H. Ohashi, S. Owada, K. Tono, M. Yabashi, E. Shigemasa, S. Yamamoto, M. Kotsugi, H. Wadati et al. Phys. Rev. Lett. 120, 223902 (2018).

[27] T. E. Glover, D. M. Fritz, M. Cammarata, T. K. Allison, S. Coh, J. M. Feldkamp, H. Lemke, D. Zhu, Y. Feng, R. N. Coffee, M. Fuchs, S. Ghimire, J. Chen, S. Shwartz, D. A. Reis, S. E. Harris, and J. B. Hastings, Nature (London) 488, 603 (2012).

[28] L. Foglia, F. Capotondi, R. Mincigrucci, D. Naumenko, E. Pedersoli, A. Simoncig, G. Kurdi, A. Calvi, M. Manfredda, L. Raimondi, N. Mahne, M. Zangrando, C. Masciovecchio, and F. Bencivenga, Phys. Rev. Lett. 120, 263901 (2018).

[29] K. Tamasaku, E. Shigemasa, Y. Inubushi, T. Katayama, K. Sawada, H. Yumoto, H. Ohashi, H. Mimura, and M. Yabashi, Nat. Photonics 8, 313 (2014).

[30] W. Hübner and K.-H. Bennemann, Phys. Rev. B 40, 5973 (1989).

[31] A. Kirilyuk, V. V. Pavlov, R. V. Pisarev, and Th. Rasing, Phys. Rev B 61, R3796 (2000).

[32] M. Fiebig, V. V. Pavlov, and R. V. Pisarev, J. Opt. Soc. Am. B 22, 96 (2005).

[33] J. Orenstein, Phys. Rev. Lett. 107, 067002 (2011).

[34] S. W. Lovesey, T. Chatterji, A. Stunault, D.?D. Khalyavin, and G. van der Laan Phys. Rev. Lett. 122, 047203 (2019).

[35] S. W. Lovesey, D. D. Khalyavin, and G. van der Laan J. Phys.: Conf. Ser. 1316, 012004 (2019).

[36] S. W. Lovesey, D. D. Khalyavin, and U. Staub, J. Phys.: Condens. Matter 27, 292201 (2015); S. W. Lovesey and D. D. Khalyavin, ibid. 27, 495601 (2015).

[37] T. Timusk and B. Statt, Rep. Prog. Phys. 62, 61 (1999).

[38] P. Bourges and Y. Sidis, C. R. Phys.12, 461 (2011).

[39] Y. Li et al., Phys. Rev. B 84, 224508 (2011).

[40] B. T. Thole, P. Carra, F. Sette, and G. van der Laan, Phys. Rev. Lett. 68, 1943 (1992).

[41] P. Carra, B. T. Thole, M. Altarelli, and X. D. Wang, Phys. Rev. Lett. 70, 694 (1993).

[42] P. Carra, H. König, B.T. Thole, and M. Altarelli, Physica B 192, 182 (1993).

[43] S. P. Collins and S. W. Lovesey, Sci. Rep. 8, 7941 (2018).

[44] S. W. Lovesey and G. van der Laan, Phys. Rev. B 98, 155410 (2018).

[45] S. W. Lovesey, J. T. Collins, and S. P. Collins, Phys. Rev. B 99, 054428 (2019).

[46] S. W. Lovesey and G. van der Laan, Phys. Rev. B 100, 245112 (2019).

[47] B. R. Judd, Phys. Rev. 127, 750 (1962). 
[48] G. S. Ofelt, J. Chem. Phys. 37, 511 (1962).

[49] S. W. Lovesey, E. Balcar, K. S. Knight, and J. F. Rodriguez, Phys. Rep. 411, 233 (2005).

[50] D. A. Varshalovich, A. N. Moskalev, and V. K. Khersonskii, Quantum Theory of Angular Momentum (World Scientific, Singapore, 1988).

[51] E. Balcar and S. W. Lovesey, Introduction to the Graphical Theory of Angular Momentum, Springer Tracts in Modern Physics Vol. 234 (Springer, Heidelberg, 2009).

[52] J. D. Byers, H. I. Yee, T. Petrallimallow, and J. M. Hicks, Phys. Rev. B 49, 14643 (1994).

[53] P. J. Brown, in International Tables for Crystallography, edited by E. Prince (Springer, Amsterdam, 2004), Vol. C, pp.454-461.
[54] S. W. Lovesey, Phys. Scr. 90, 108011 (2015).

[55] S. W. Lovesey, Theory of Neutron Scattering from Condensed Matter (Clarendon, Oxford, 1987), Vol. 2.

[56] S. W. Lovesey and E. Balcar, J. Phys. Soc. Jpn. 79, 074707 (2010); 79, 104702 (2010).

[57] S. W. Lovesey and G. van der Laan, Phys. Rev. B 101, 144419 (2020).

[58] D. D. Khalyavin and S. W. Lovesey, Phys. Rev. B 100, 224415 (2019).

[59] A. Abragam and B. Bleaney, Electron Paramagnetic Resonance of Transition Ions (Clarendon, Oxford, 1970).

[60] S. W. Lovesey, J. Phys.: Condens. Matter 26, 356001 (2014). 